\title{
Physical Properties and Light-Related Applications of Black Silicon Structures
}

Emil Pincik ${ }^{1 *}$, Hikaru Kobayashi ${ }^{2}$, Róbert Brunner ${ }^{1}$, Kentaro Imamura ${ }^{2}$, Milan Mikula ${ }^{3}$, Michal Kucera ${ }^{4}$, Pavel Vojtek $^{5}$, Zuzana Zabudla ${ }^{5}$, PeterSvec $\mathrm{Sr}^{1}$, Ján Gregus ${ }^{5}$ and PeterSvec $\mathrm{Jr}^{1}$

1. Institute of Physics, SAS, Dubravska cesta 9, 84511 Bratislava, Slovak Republic

2. Institute of Scientific and Industrial Research, Osaka University and CREST, 8-1 Mihogaoka, Ibaraki, Osaka 567-0047, Japan

3. Faculty of Chemical and Food Technology of STU, Radlinského 9, 81237 Bratislava, Slovak Republic

4. Institute of Electrical Engineering SAS, Dubravska cesta 9, 84104 Bratislava, Slovak Republic

5. Faculty of Mathematics, Physics, and Informatics of Comenius University, Mlynska Dolina, F2, 84248 Bratislava, Slovak Republic

\begin{abstract}
This contribution deals with the black silicon (BS) nanocrystalline specimens produced using the surface structure chemical transfer method (SSCT). This method can produce a nanocrystalline Si black color layer on c-Si with a thickness range of $\sim 50 \mathrm{~nm}$ to $\sim 300 \mathrm{~nm}$ via the contact of $\mathrm{c}-\mathrm{Si}$ immersed in the chemical solution $\mathrm{HF}+\mathrm{H}_{2} \mathrm{O}_{2}$ with a catalytic mesh. The photoluminescence properties are related to the formation of nanocrystals, the structural properties of which are similar to those formed on the back of a sawn Si wafer and the resulting splitting of large Si crystals. X-ray diffraction of the Si front and back sides confirms the dominant reflection of the $311 \mathrm{Si}$ crystalline planes. We suppose that the formation of the black silicon over-layer is pre-determined by the crystalline defects induced by the applied sawing procedure, even though saw damage (defects introduced by the applied sawing procedure) is not necessary for the SSCT method. The formation of the pn type Si solar cell is presented, including black silicon over-layer and without antireflection coating, with efficiency of $\sim 19.1 \%$.
\end{abstract}

Key words: Photoluminescence, black silicon, solar cell.

\section{Introduction}

Solar cell structures need low reflectance surfaces to maximize the number of absorbed incident photons, with antireflection (AR) coatings commonly being used in the solar cell industry. However, the use of such coatings is limited because they reduce reflection for a narrow range of light wavelengths [1,2]. Jansen et al. [3] firstly demonstrated the replacement of conventional AR coatings with a black silicon layer. Nanocrystalline black Si samples can be produced using multiple methods [4, 5].

Nanocrystalline black $\mathrm{Si}$ is formed by an electrochemical reaction from the surface, with a nanocrystalline Si layer forming on the surface. The used chemical solution penetrates the space region of

*Corresponding author: Emil Pincik, PhD., research fields: development of solar cells, semiconductor physics and technology. the nanocrystalline Si layer, leading to the growth of the nanocrystalline $\mathrm{Si}$ layer. With this formation mechanism a low reflectivity nanocrystalline Si layer is formed. The low reflectivity is probably caused by the special depth profile of the refractive index, which is close to 1 near the surface and increases with depth. We suppose that the space/Si volume ratio in the nanocrystalline Si layer is high near the surface and decreases with depth. In the case of the multicolor wafer, the space/Si volume ratio seems to be low even near the surface, leading to relatively high reflectivity of $\sim 10 \%$.

$\mathrm{Si}$ nanocrystallites embedded in $\mathrm{SiO}_{2}$ thin films were investigated by Ding et al. [6]. Observed photoluminescence (PL) spectra demonstrated a continuous annealing temperature-based evolution. In dependence on the annealing temperature, six PL bands located at 415, 460, 520, 630, 760 and $845 \mathrm{~nm}$, 
respectively, have been observed, whereby annealing at $1,100{ }^{\circ} \mathrm{C}$ yields the strongest PL band at $760 \mathrm{~nm}$. Wong et al. [7] observed significantly enhanced PL bands at $650 \mathrm{~nm}$ and $750 \mathrm{~nm}$ in a similar structure (Si nanocrystals in $\mathrm{SiO}_{2}$ ) after high temperature annealing at $1,100{ }^{\circ} \mathrm{C}$. They explained this effect by the formation of Si nanocrystallites via a phase separation reaction, and by the removal of defect-related absorption centers during annealing. Kuznetsov et al. [8] investigated the PL properties of an array of vertically aligned $\mathrm{Si}$ nanopillars with average diameters of $\sim 100 \mathrm{~nm}$ and $\sim 5 \mu \mathrm{m}$ length. A strong broad visible PL emission band around $1.8 \mathrm{eV}$, with a nearly perfect Gaussian shape, was observed in porous-walled nanopillars - Si nanocrystals passivated with $\mathrm{SiO}_{(\mathrm{x})}$. The authors found the $\mathrm{Si}$ nanocrystal surface most probably to be responsible for the luminescence. The red shift of the emission maximum and the luminescence quenching induced by oxidation of the samples in the UV ozone partly confirmed this assumption. A model of luminescence has been proposed involving UV photon absorption by Si nanocrystals with a subsequent exciton radiative recombination on defect sites in $\mathrm{SiO}_{(\mathrm{x})}$ covering the $\mathrm{Si}$ nanocrystals.

Lü et al. [9] observed a PL band with a maximum in the range $0.78-0.84 \mathrm{eV}$ on black Si structures when the annealing temperature changed from $500{ }^{\circ} \mathrm{C}$ to $1,200{ }^{\circ} \mathrm{C}$, respectively. Goshi et al. [10] investigated the origin of the visible and near infrared PL of chemically etched $\mathrm{Si}$ nanowires adorned with arbitrarily shaped $\mathrm{Si}$ nanocrystals. Their studies revealed that Si nanocrystals are primarily responsible for the emission in the $1.5-2.2 \mathrm{eV}$ range, in dependence on the cross-section of the $\mathrm{Si}$ nanocrystals. The large diameter $\mathrm{Si} / \mathrm{SiO}_{(\mathrm{x})}$ nanowires yield a distinct NIR PL signal that consists of peaks at 1.07, 1.10 and $1.12 \mathrm{eV}$. The latter NIR peaks were attributed to the room temperature $\mathrm{TO} / \mathrm{LO}$ phonon-assisted radiative recombination of free carriers condensed in the electron-hole plasma in etched Si nanowires. The enhancement of PL intensity and the red shift of the PL peak measured at low temperatures were explained as a consequence of the interplay of radiative and non-radiative recombinations at the interfaces of Si nanocrystals and $\mathrm{Si} / \mathrm{SiO}_{(\mathrm{x})}$ nanowires. Researchers at ISIR Osaka University developed a fabrication method of low reflectivity Si surfaces in which a mold with catalytic Pt layer is placedinto contact with $\mathrm{Si}$ immersed in a $\mathrm{H}_{2} \mathrm{O}_{2}+\mathrm{HF}$ solution [11]. The energy of PL maximum observed for the black silicon layer prepared on a polycrystalline $\mathrm{Si}$ wafer at room temperature was $\sim 1.85 \mathrm{eV}$ [12]. A similar method was also applied for the preparation of the OBSi samples in this contribution. An additional alternative technological approach was developed in the same laboratories in Japan by M. Takahashi et al. [13].

In this contribution we will present and discuss the optical properties of both a black silicon sample and the front and back sides of the reference Si sample (without RCA treatment), respectively, and the properties of solar cell structures prepared both with and without black silicon over-layer.

\section{Materials}

Black silicon nanocrystalline specimens were produced sing the surface structure chemical transfer method. This method can produce a nanocrystalline $\mathrm{Si}$ layer on c-Si with a thickness of $\sim 50 \mathrm{~nm}$ (multi-color) to $\sim 300 \mathrm{~nm}$ (black color) by the contact of $\mathrm{c}-\mathrm{Si}$ immersed in chemical solutions of $\mathrm{HF}+\mathrm{H}_{2} \mathrm{O}_{2}$ with a catalytic mesh.

In the case of the SSCT method, laboratories of the ISIR Osaka University in Japan can produce ultralow reflectivity surfaces using mirror-like polished $\mathrm{Si}$ wafers (for LIS use), and therefore, defects are not necessary for the production of a nanocrystalline $\mathrm{Si}$ layer. The SSCT method forms a nanocrystalline $\mathrm{Si}$ layer with the following electrochemical reaction:

Decomposition of $\mathrm{H}_{2} \mathrm{O}_{2}$ on $\mathrm{Pt}$ to generate positive charges:

$$
\mathrm{H}_{2} \mathrm{O}_{2}+\mathrm{H}^{+} \rightarrow \mathrm{H}_{2} \mathrm{O}+\mathrm{OH}^{-}+2 h
$$


Positive charges are injected to $\mathrm{Si}$, diffuse to $\mathrm{Si}$ surfaces, and holes (h) are captured at surface states, e.g., Si dangling bond states. In the presence of holes, $\mathrm{Si}$ strongly reacts with $\mathrm{HF}$, resulting in dissolution of Si.

$$
\mathrm{Si}+6 \mathrm{HF} \rightarrow \mathrm{H}_{2} \mathrm{SiF}_{6}+\mathrm{H}_{2}+2 \mathrm{H}^{+}
$$

Because surface states are present inhomogeneously, nonuniform Si dissolution occurs, resulting in the formation of the nanocrystalline $\mathrm{Si}$ layer. Acid etching used for texturing of polycrystalline $\mathrm{Si}$ wafers proceeds from saw damage, and therefore, on polycrystalline $\mathrm{Si}$ wafers produced using the fixed abrasive technology without high density saw damage defects, acid etching cannot form textured structure while the SSCT method can form ultralow reflectivity surface from polycrystalline $\mathrm{Si}$ wafers produced with the fixed abrasive technology. This is another evidence that saw damage (defects introduced by the applied sawing procedure) is not necessary for the SSCT method.

All black silicon samples were prepared on p-type (100) Si substrates after RCA cleaning, and were stabilized with thermal oxidation at approx. $900 \mathrm{C}$ for a few minutes. Hence the abbreviation OBSi is used. The optical properties of the reference Si sample after polishing procedures and brief etching in only vapor $\mathrm{HF}$ are also investigated. The corresponding abbreviation is Ref Si.

The form of catalytic mesh and the technology of OBSinano-structure preparation are illustrated in Fig. 1.

\subsection{Used Experimental Methods}

\subsubsection{Leica DM2700}

Standard optical microscope was used to determine the morphology of treated surfaces and/or back sides of the silicon structures. The AFM records were obtained with VEECO CP2 equipment. Tip radius of $10 \mathrm{~nm}$ and a contact force of $10 \mathrm{nN}$ were used in contact mode.

\subsubsection{Digilab Excalibur FTS 3000 MX}

The spectrometer was used for FTIR spectroscopy with the following FTIR spectroscopy software: ResolutionsPro 5.2.0, Agilent with Kramers-Kronig transformation. A PIKE Technologies Specular Reflectance Accessory was used at an angle of $30^{\circ}$. The measured area's diameter was $10 \mathrm{~mm}$, used resolution: $4 \mathrm{~cm}^{-1}$, number of scans: 60 .

\subsubsection{Bruker D8 Advance}

$\mathrm{X}$-ray diffraction at grazing incidence angles has been measured with this equipment, with a $60 \mathrm{~mm}$ Goebel mirror for $\mathrm{Cu}$ radiation and $5^{\circ}$ Soller slit in the primary beam path. The secondary beam path was fitted with a $0.23^{\circ}$ parallel plate collimator and $\mathrm{LiF}$ monochromator to enhance instrument resolution. The emitted beam shape was $10 \mathrm{~mm} \times 1.2 \mathrm{~mm}$.

\subsubsection{Photoluminescence (PL)}

The measurements were performed at room and $6 \mathrm{~K}$ temperatures with equipment that used a halogen lamp as the light source, and a PL spectrum in the range 420-1,000 nm was detected by the CCD element.

\section{Results and Discussion}

\subsection{Optical and X-Ray Methods}

The photoluminescence records of Fig. 2 were recorded on both Ref Si (surface side after polishing and cleaning with HF vapor) and OBSi, respectively, with PL apparatus with a focusing system and cooled Si photodetector. These records illustrate the blue shift of the PL spectra by approx. $0.40 \mathrm{eV}$, with the change of the sample temperature from room one to $6 \mathrm{~K}$. During the PL measurements, the OBSi sample was hung outside the metallic sample holder to avoid any potential influence of the supporting material on the PL spectra form. The results of fitting procedures are

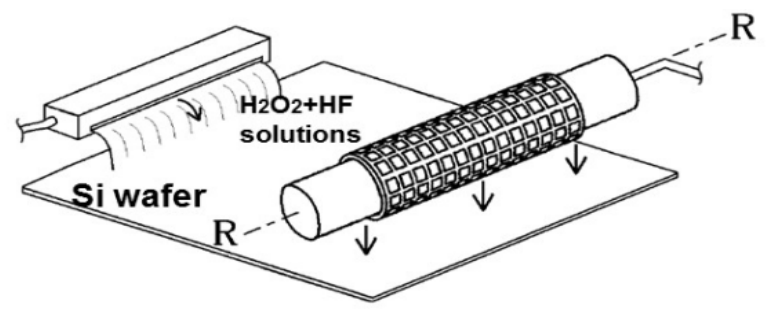

Fig. 1 The form of catalytic mesh and the technology of OBSinano-structure preparation. 


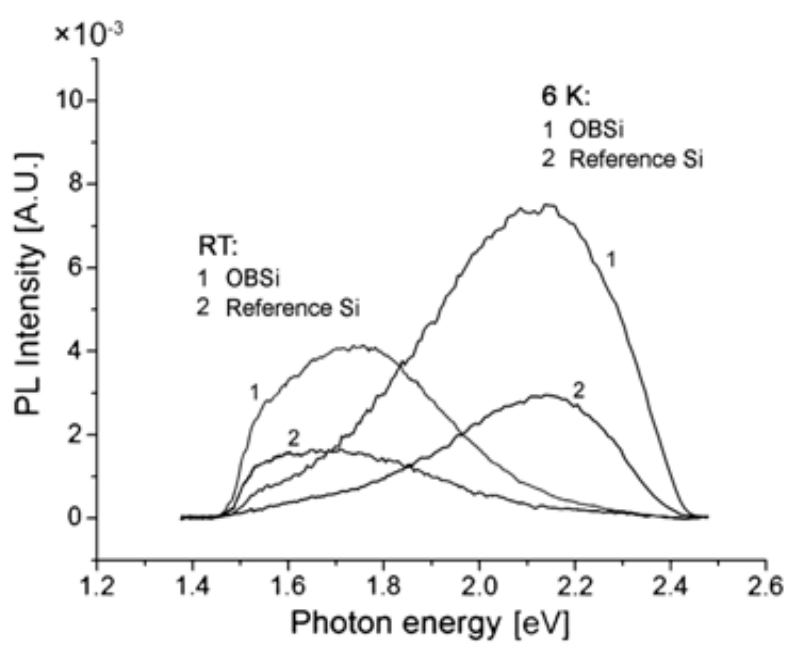

Fig. 2 Photoluminescence signals recorded at room temperature and $6 \mathrm{~K}$ on both Ref Si (surface side after polishing and cleaning with $\mathrm{HF}$ vapor) and the OBSi.

presented in Table 1, Table 2 and Table 3. Three peaks fitting Gaussian curves have been optimal for these structures due to the lowest value of residuum. The corresponding analytical procedure has been described in detail in Ref. [14].

On the top and back sides of the reference $\mathrm{Si}$ sample after the polishing procedures and HF etching (removing $\mathrm{SiO}_{2}$ native oxide), photoluminescence signals were also recorded at room temperature. The $\mathrm{PL}$ record of the reference $\mathrm{Si}$ back side is shown in Fig. 3. The absolute value of the PL signal was higher than that observed on the OBSi by approximately two orders. The data obtained with the fitting procedure are presented in Table 4. The morphology of the reference Si back side is shown in Fig. 4 obtained with an optical microscope. Fig. 5 shows the surface of the Si back side obtained by the AFM. The roughness of the sample is $\sim 127 \mathrm{~nm}$. Fig. 6 presents the FTIR reflectance spectra of both the front and back side of the reference Si sample obtained with a diamond tip. Dispersion dependences of $n$ and $k$ on the wavenumber of both investigated Ref Si regions (front and back sides) were calculated from the IR reflectance measurements at $30^{\circ}$ - see Figs. 7 and 8, respectively. Standard commercial software was used. Prior to RCA cleaning, the thickness of the structurally disturbed Si surface region resting after the
Table 1 Data obtained by fitting procedures of PL records of the front side of the $\mathrm{Si}$ reference sample at room temperature (a) and at $6 \mathrm{~K}$ (b).

\begin{tabular}{llll}
\hline No. & Energy (eV) & Intensity (A.U.) & FWHM (eV) \\
\hline 1 & 1.57 & $7.76 \mathrm{e}-4$ & 0.11 \\
2 & 1.70 & $1.04 \mathrm{e}-3$ & 0.24 \\
3 & 1.89 & $7.53 \mathrm{e}-4$ & 0.40 \\
Offset & $2.68 \mathrm{e}-5$ & (A.U.) & \\
Residuum & $1.58 \mathrm{e}-3$ & (A.U.) & \\
\hline \multicolumn{4}{r}{ (b) } \\
\hline No. & Energy (eV) & Intensity (A.U.) & FWHM (eV) \\
\hline 1 & 1.91 & $3.38 \mathrm{e}-3$ & 0.45 \\
2 & 2.10 & $4.95 \mathrm{e}-3$ & 0.29 \\
3 & 2.27 & $3.11 \mathrm{e}-3$ & 0.19 \\
Offset & 0 & (A.U.) & \\
Residuum & $5.27 \mathrm{e}-3$ & (A.U.) & \\
\hline
\end{tabular}

Table 2 Data obtained by fitting procedures of PL records of the front side of the OBSi at room temperature (a) and at $6 \mathrm{~K}$ (b).

(a)

\begin{tabular}{llll}
\hline No. & Energy (eV) & Intensity (A.U.) & FWHM (eV) \\
\hline 1 & 1.57 & $1.44 \mathrm{e}-3$ & 0.11 \\
2 & 1.71 & $2.48 \mathrm{e}-3$ & 0.24 \\
3 & 1.88 & $2.13 \mathrm{e}-3$ & 0.37 \\
Offset & $2.56 \mathrm{e}-5$ & (A.U.) & \\
Residuum & $2.93 \mathrm{e}-3$ & (A.U.) & \\
\hline \multicolumn{4}{r}{} \\
\hline No. & Energy (eV) & Intensity (A.U.) & FWHM (eV) \\
\hline 1 & 1.86 & $8.78 \mathrm{e}-4$ & 0.44 \\
2 & 2.09 & $2.21 \mathrm{e}-3$ & 0.29 \\
3 & 2.24 & $1.18 \mathrm{e}-3$ & 0.19 \\
Offset & 0 & (A.U.) & \\
Residuum & $1.44 \mathrm{e}-3$ & (A.U.) & \\
\hline
\end{tabular}

Table 3 Values of the blue shift of fitted photoluminescence spectra with the change of the sample temperature from room one to $6 \mathrm{~K}$. The OBSi sample has been hanged near the sample support.

(a)

Ref I (6 K) - Ref I (RT)

\begin{tabular}{llll}
\hline No. & Energy $(\mathrm{eV})$ & Energy $(\mathrm{eV})$ & Energy $(\mathrm{eV})$ \\
\hline 1 & 1.91 & 1.57 & 0.34 \\
2 & 2.10 & 1.70 & 0.40 \\
3 & 2.27 & 1.89 & 0.38 \\
\hline
\end{tabular}

(b)

\begin{tabular}{llll}
\multicolumn{2}{l}{ OBSi $(6 \mathrm{~K})$} & OBSi $(\mathrm{RT})$ \\
\hline No. & Energy $(\mathrm{eV})$ & Energy $(\mathrm{eV})$ & Energy $(\mathrm{eV})$ \\
\hline 1 & 1.86 & 1.57 & 0.29 \\
2 & 2.09 & 1.71 & 0.38 \\
3 & 2.24 & 1.88 & 0.36 \\
\hline
\end{tabular}




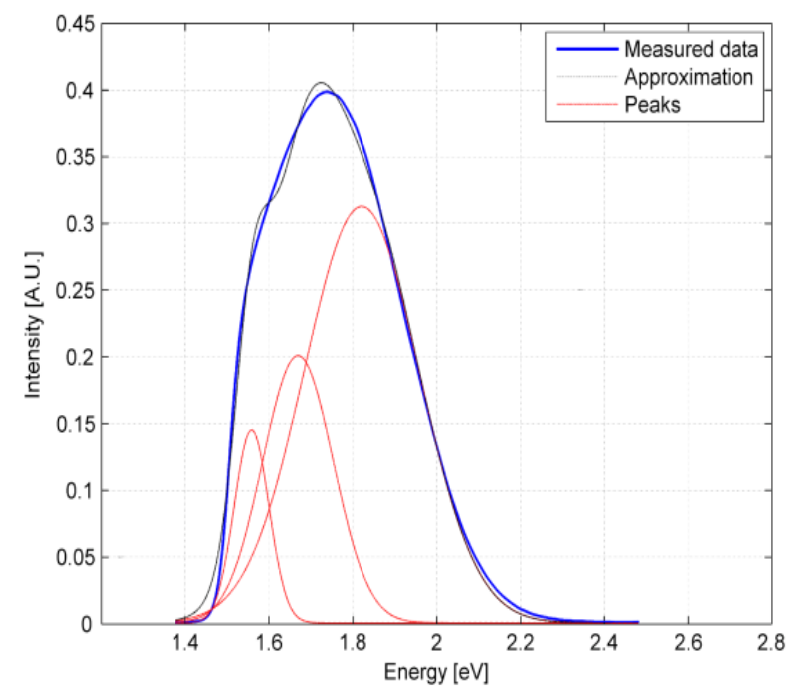

Fig. 3 Photoluminescence of the reference Si back side recorded at room temperature.

Table 4 Data obtained by fitting procedures of PL records recorded at room temperature of the back side of the $\mathrm{Si}$ reference sample.

\begin{tabular}{llll}
\hline No. & Energy (eV) & Intensity (A.U.) & FWHM (eV) \\
\hline 1 & 1.56 & $1.45 \mathrm{e}-1$ & 0.10 \\
2 & 1.67 & $2.00 \mathrm{e}-1$ & 0.20 \\
3 & 1.82 & $3.12 \mathrm{e}-4$ & 0.32 \\
Offset & $7.41 \mathrm{e}-4$ & (A.U.) & \\
Residuum & $3.14 \mathrm{e}-1$ & (A.U.) & \\
\hline
\end{tabular}

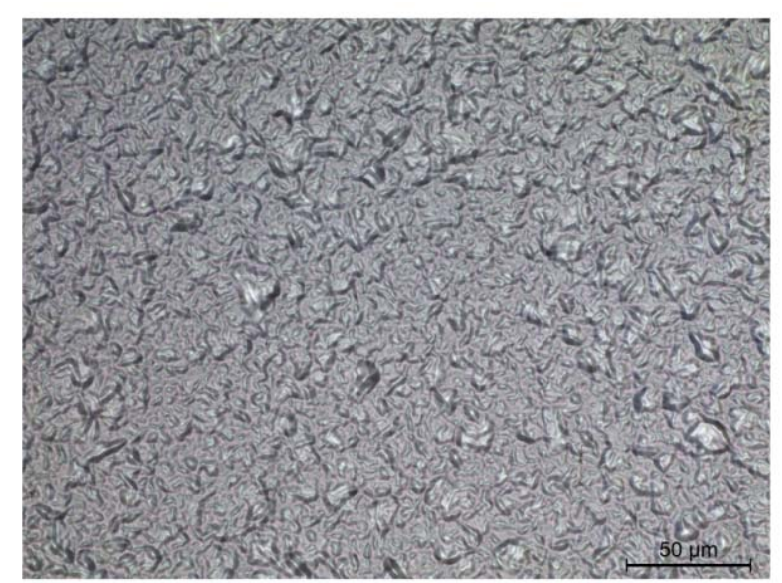

Fig. 4 The morphology of the reference Si back side obtained with optical microscope.

polishing procedure is $\sim 100 \mathrm{~nm}$. This damaged region also contains broken surface parts of the c-Si wafer of varying sizes covered with native $\mathrm{SiO}_{(\mathrm{x})}$. The Si back side contains larger residues of crystalline parts of the original Si crystal. Fig. 9 shows the X-ray diffraction pattern of the Ref $\mathrm{Si}$ back side recorded at the following three grazing incidence angles $-1^{\circ}, 2^{\circ}$ and $3^{\circ}$. Reflection 311 dominates. A comparison of the XRDGI patterns of Fig. 9 and those presented in [15]

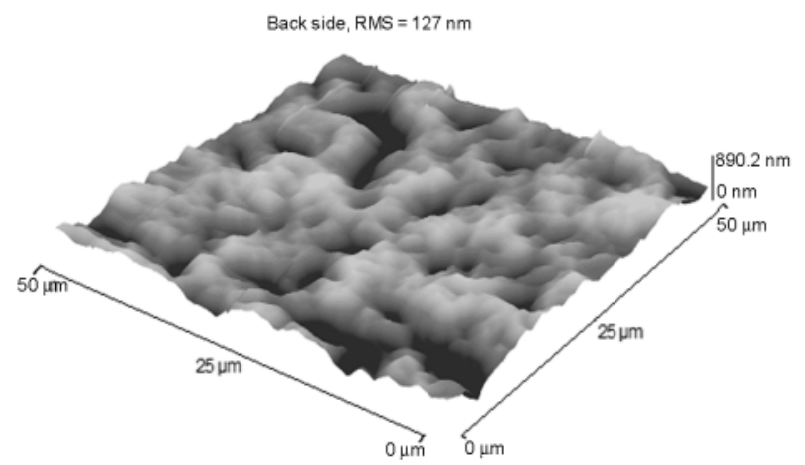

Fig. 5 The surface of the Si back side obtained by AFM.

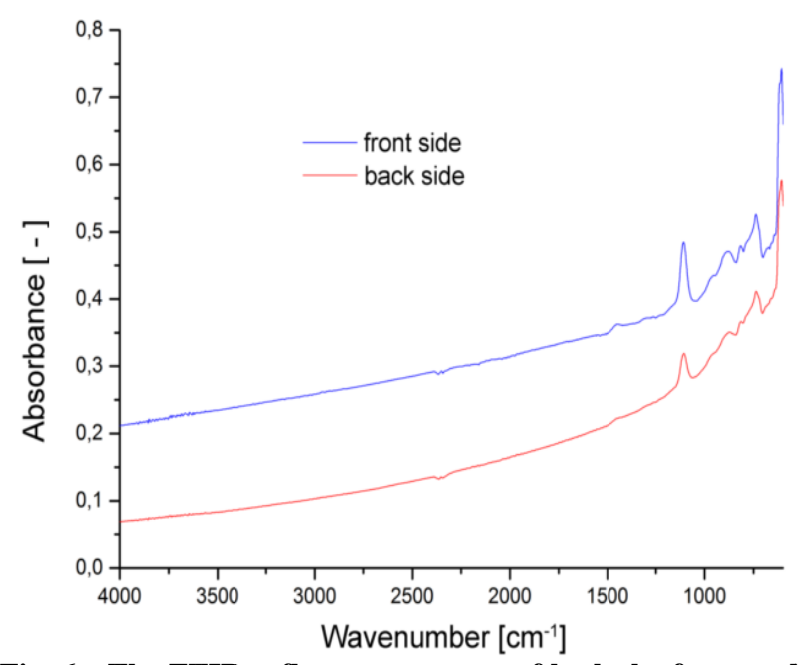

Fig. 6 The FTIR reflectance spectra of both the front and back side of the reference $\mathrm{Si}$ sample obtained with a diamond tip.

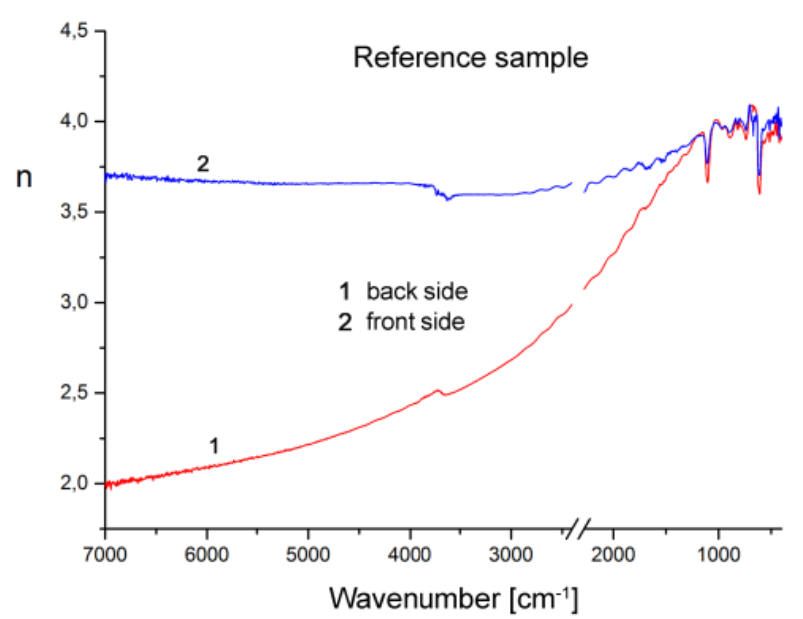

Fig. 7 Dispersion dependences of $\boldsymbol{n}$ on the wavenumber of the front and back sides of the reference Si after polishing procedures. 


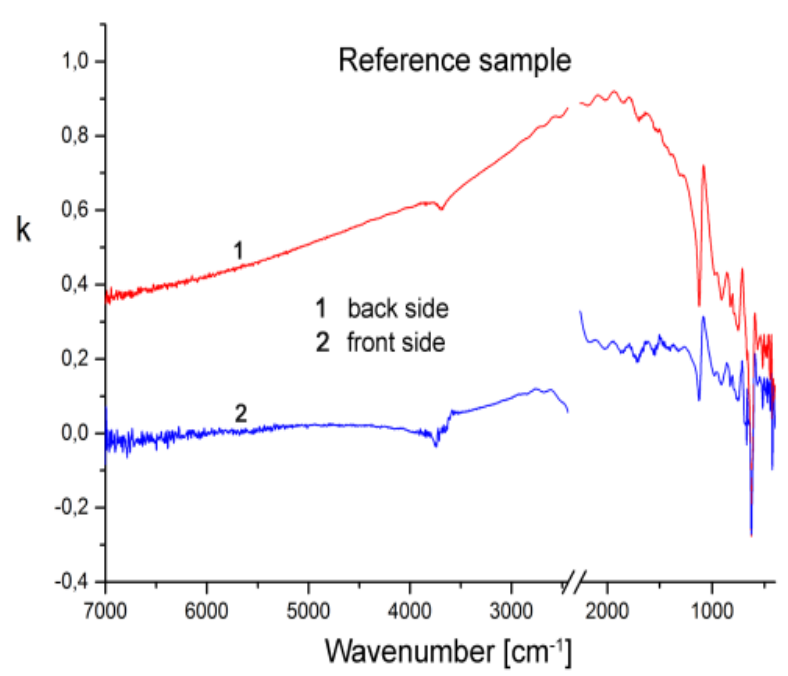

Fig. 8 Dispersion dependences of $\boldsymbol{k}$ on the wavenumber of the front and back sides of the reference Si after polishing procedures.

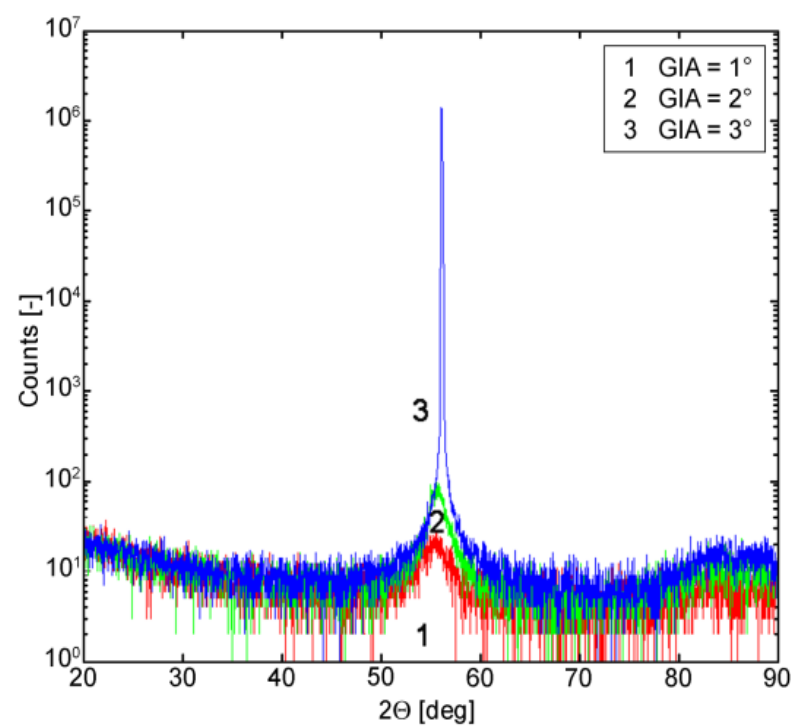

Fig. 9 X-ray diffraction patterns of the Ref Si back side recorded at the following three grazing incidence angles $1^{\circ}, 2^{\circ}$ and $3^{\circ}$. Reflection 311 dominates.

confirm that the XRDGI records of all structures (OBSi, Ref Si front and back sides) are very similar, in fact almost identical. The dominant difference is given by the absolute value of structures' intensities. In comparison with the results obtained on the porous $\mathrm{Si}$ and published in [16], a stronger signal of reflection 111 is missing in the case of all investigated samples.

One of the determining parameters influencing the absolute intensity value of the recorded PL signals in excited optical regions of the front and back sides of the Ref Si sample can be related to: i) the illuminated size of the sample areas, and ii) the nanocrystalline structure of the illuminated Si side.

Recorded PL signals are related to the formation of nanocrystals, the structural properties of which are similar to those formed on the back side of the $\mathrm{Si}$ wafer after the large Si crystal has been sawn. X-ray diffraction of the Si front and back sides confirms the dominant reflection of the $311 \mathrm{Si}$ crystalline planes. We suppose that the formation of the black silicon over-layer is pre-determined by crystalline

Recorded PL signals are related to the formation of nanocrystals the structural properties of which are similar to those formed on the back side of the $\mathrm{Si}$ wafer after the large Si crystal has been sawn. X-ray diffraction of the Si front and back sides confirms the dominant reflection of the $311 \mathrm{Si}$ crystalline planes. We suppose that the formation of the black silicon over-layer is pre-determined by crystalline defects induced by the used sawing procedure. Both reference Si sides have very similar PL spectra, even after their deconvolution to three separate Gaussians.

It needs to be stated that correct photoluminescence (PL) signals in the photon energy region $1.3-2.5 \mathrm{eV}$ were not recorded in the polished Si structure after RCA cleaning.

The blue shift of both the PL spectra (reaching almost a value of $0.40 \mathrm{eV}$ (see Table 3) observed after the decrease of sample temperature to approx. $6 \mathrm{~K}$, we also relate to the change of semiconductor band gap width, i.e. with the change of semiconductor properties of structures generating PL light, with the amorphous structure containing $\mathrm{Si}$ nanocrystalline grains of the OBSi and the front reference Si surface regions on which the additional effect - quantum confinement - is probably observed. The band gap width of semiconductors typically decreases with increasing temperature, see e.g. [17], due to the change of electron-phonon interaction and the expansion of the lattice. We suppose that in the case of a higher concentration of defect states at the 
$\mathrm{SiO}_{2} / \mathrm{Si}$ interface also the form of tails of conduction and valence bands can be strongly changed - reduced by the change of sample temperature of room one to approx. $6 \mathrm{~K}$.

In agreement with our conclusions in the paper [15], we suppose that the PL bands recorded at room temperature between $1.70 \mathrm{eV}$ and $1.90 \mathrm{eV}$ on both Ref I Si and OBSi can be related to the defects formed at the $\mathrm{SiO}_{(\mathrm{x})} / \mathrm{Si}$ interface region of both structures during their preparation. Our results at least partly confirm, the conclusions of Kuznetsov et al. [8] - the model of luminescence involving UV photon absorption by $\mathrm{Si}$ nanocrystals with subsequent exciton radiative recombination on defect sites in $\mathrm{SiO}_{(\mathrm{x})}$ covering $\mathrm{Si}$ nanocrystals can also be proposed.

Of course, it is necessary to state that broad PL records are not complete because of the above-mentioned two limits determined by both the used monochromator and photodetector, respectively. This fact complicates the precise evaluation and discussion of PL results. Very similar PL behaviors recorded at liquid helium $(6 \mathrm{~K})$ and room temperatures on both polished crystalline $\mathrm{Si}$ and OBSi samples, respectively, indicate the similar origin of recorded PL bands. As the positions of PL maxima of OBSi structures are mainly related to the size of $\mathrm{Si}$ nanocrystallites and $\mathrm{SiO}_{(\mathrm{x})}$, we therefore suppose that the size of dominant parts of the luminated OBSi nanostructure is pre-determined by the used cutting and polishing $\mathrm{Si}$ procedure, and/or the distribution functions of the number of nanocrystallites (formed by cutting and polishing procedures and by the SSCT method) on their size is very similar. It can be supposed that the electrochemical catalytic process of the formation of nanocrystallites using the SSCT method starts along the nanocrystalline grain boundaries residing in the Si surface region after the
RCA cleaning procedure.

\subsection{Solar Cells}

On the Si sample with $\sim 150 \mathrm{~nm}$ thickness of black silicon over-layer, a corresponding pn junction was formed - see Fig. 10.

The solar cell parameters (I-V curves) of structures with the BS are compared with a solar cell formed with the same technological steps on the Si substrate without BS over-layer - see Fig. 11. The solar cell parameters of both structures were measured using standard commercial equipment - the results are shown in Table 5 .

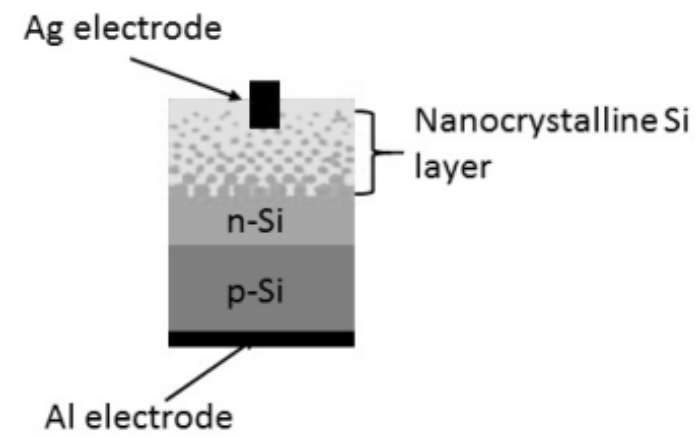

Fig. 10 Illustration of the pn junction formed on the black silicon structure.

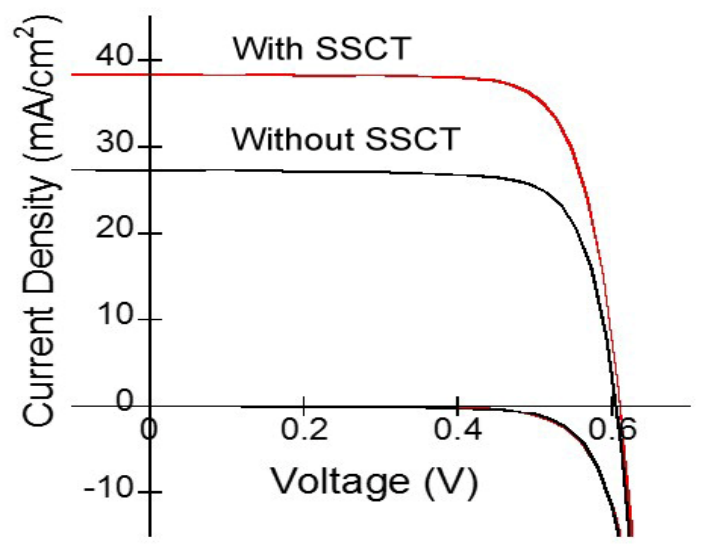

Fig. 11 Solar cell parameters (I-V curves) of structures with the black silicon over-layer are compared with the solar cell formed by the same technological steps on the $\mathrm{Si}$ substrate without the over-layer.

Table 5 Solar cell parameters of both structures - with and without black silicon over-layer.

\begin{tabular}{lllll}
\hline & $\mathrm{V}_{\mathrm{OC}}(\mathrm{V})$ & $\mathrm{J}_{\mathrm{SC}}\left(\mathrm{mA} / \mathrm{cm}^{2}\right)$ & $\mathrm{FF}$ & Conversion effic. (\%) \\
\hline With SSCT & 0.608 & 40.8 & 0.771 & 19.1 \\
Without SSCT & 0.601 & 27.3 & 0.771 & 12.7 \\
\hline
\end{tabular}




\section{Conclusions}

This contribution deals with the photoluminescence of both i) oxidized black silicon (OBSi) nano-crystalline specimens produced by the surface structure chemical transfer method (SSCT), and ii) both sides of a polished reference $\mathrm{Si}$ sample, respectively. The similar PL behaviors recorded at liquid helium and room temperatures on both the polished crystalline Si surface and the OBSi samples, respectively, indicate the similar origin/similar sources of the recorded luminescence light. The absolute value of the PL signal was higher than that observed on the OBSi by approximately two orders. As the positions of PL maxima of all structures are mainly related to the size of $\mathrm{Si}$ nanocrystallites and $\mathrm{SiO}_{(\mathrm{x})}$, then we suppose that the size of the dominant luminated parts of all structures is pre-determined by the used cutting and polishing Si procedure even though saw damage (defects introduced by the applied sawing procedure) is not necessary for the SSCT method, and/or the distribution functions of the number of nanocrystallites (formed by the cutting and polishing procedures and the SSCT method) on their size is very similar. It can be supposed that the electrochemical catalytic process of the formation of nanocrystallites with the SSCT method starts along the $\mathrm{Si}$ nanocrystalline grain boundaries that reside in the $\mathrm{Si}$ surface region after RCA cleaning. The blue shift of both the PL spectra, almost reaching a value of $0.40 \mathrm{eV}$ and observed after the decrease of the sample temperature to $6 \mathrm{~K}$, we relate to the change of semiconductor band gap width, i.e. the change of semiconductor properties of structures that generate the PL light.

Our model of observed photoluminescence phenomena involves UV photon absorption by $\mathrm{Si}$ nanocrystals with subsequent exciton radiative recombination on defect sites in $\mathrm{SiO}_{(\mathrm{x})}$ covering $\mathrm{Si}$ nanocrystals. The model is valid for photoluminescence observed on both the black silicon structures and the reference Si (front and back sides), respectively.

We have shown that the efficiency of the pn-type $\mathrm{Si}$ solar cell with the black silicon overlayer can reach $19.1 \%$ without antireflection coating being used.

\section{Acknowledgements}

This work was partly financially supported by the Japan Society for the Promotion of Science (JSPS) and by the following agencies: APVV - Project No. APVV-15-0152 and VEGA - projects Nos. 2/0076/15 and $1 / 0900 / 16$.

\section{References}

[1] Ma, L. L., Zhou, Y. C., Jiang, N., Lu, X., Shao, J. and Lu, W. et al. 2006. "Wide-Band 'Black Silicon' Based on Porous silicon.” Appl. Phys. Lett. 88: 171907.

[2] Walheim, S., Schaffer, E., Mlynek, J. and Steiner, U. 1999. "Structure Formation via Polymer Demixing in Spin-Cast Films." Science 283: 520-2.

[3] Jansen, H., de Boer, M., Legtenberg, R. and Elwenspoek, M. 1995. "The Black Silicon Method: A Universal Method for Determining the Parameter Setting of a Fluorine-Based Reactive Ion Etcher in Deep Silicon Trench Etching with Profile control." J. Micromech. Microeng. 5: 115-20.

[4] Ayon, A. A., Braff, R., Lin, C. C., Sawin, H. H. and Schmidt, M. A. 1999. "Characterization of a Time Multiplexed Inductively Coupled Plasma Etcher." $J$. Electrochem. Soc. 146: 339-49.

[5] Wu, C., Crouch, C. H., Zhao, L., Carey, J. E., Younkin, R. and Levinson, J. A. et al. 2001. "Near-Unity Below-Band -Gap Absorption by Microstructured Silicon.” Appl. Phys. Lett. 78: 1850-2.

[6] Ding, L., Chen, T. P., Liu, Y., Ng, C. Y., Yang, M. and Wong, J. I. et al. 2008. "Evolution of Photoluminescence Mechanisms of $\mathrm{Si}+$-Implanted $\mathrm{SiO}_{2}$ Films with Thermal Annealing." J. Nanosci. Nanotechnol. 8: 3555.

[7] Wong, C. K., Wong, H. and Filip, V. 2009. "Photoluminescence of Silicon Nanocrystals Embedded in Silicon Oxide." J. Nanosci. Nanotechnol. 9 (Feb.): 1272-6.

[8] Kuznetsov, A. S., Shimizu, T., Kuznetsov, S. N., Klekachev, A. V., Shingubara, S. and Vanacken, J. et al. 2012. "Origin of Visible Photoluminescence from Arrays of Vertically Arranged Si-Nanopillars Decorated with Si-Nanocrystals." Nanotechnology 23 (Nov. 1): 475709. 
[9] Lü, Q., Wang, J., Liang, C., Zhao, L. and Jiang, Z. 2013. "Strong Infrared Photoluminescence from Black Silicon Made with Femtosecond Laser Irradiation.” Opt. Lett. 38 (April): 1274-6.

[10] Ghosh, R., Giri, P. K., Imakita K. and Fujii, M. 2014. "Origin of Visible and Near-Infrared Photoluminescence from Chemically Etched Si Nanowires Decorated with Arbitrarily Shaped Si Nanocrystals." Nanotechnology 25 (Jan): 045703.

[11] Fukushima, T., Ohnaka, A., Takahashi, M. and Kobayashi, H. 2011. "Fabrication of Low Reflectivity Poly-Crystalline Si Surfaces by Structure Transfer Method." Electrochem. Solid-State Lett. 14: B13-B15.

[12] Imamura, K., Franco Jr., F. C., Matsumoto, T. and Kobayashi, H. 2013. "Ultra-Low Reflectivity Polycrystalline Silicon Surfaces Formed by Surface Structure Chemical Transfer Method." Appl. Phys. Lett. 103: 013110.

[13] Takahashi, M., Fukushima, T., Seino, Y., Kim, W.-B., Imamura, K. and Kobayashi, H. 2013. "Surface Structure
Chemical Transfer Method for Formation of Ultralow Reflectivity Si Surfaces.” J. Electrochem. Soc. 160 (8): H443-H445.

[14] Brunner, R., Pinčík, E., Kobayashi, H., Kučera, M., Takahashi, M. and Rusnák, J. 2010. "On Photoluminescence Properties of a-Si:H-Based Structures.” Appl. Surf. Sci. 256: 5591-6.

[15] Pincik, E., Brunner, R., Kobayashi, H., Mikula, M., Vojtek, P. and Greguš, J. et al. 2016. "The Photoluminescence of Multicolor Silicon." Journal of the Chinese Advanced Materials Society 4 (April): 158-71.

[16] Pincik, E., Jergel, M., Bartos, J., Falcony, C. and Kucera, M. 1999. "The C-V Investigation of Light-Related Properties of Porous Silicon/Crystalline Silicon Structure." Superficies y Vacio 9: 78.

[17] Grundman, M. 2010. The Physics of Semiconductors, An Introduction Including Nanophysics and Applications. XXXVII, 864 pages, ISBN: 978-3- 642-13 883-6, Springer-Verlag, Berlin. 